\title{
Important role of myocardial tissue characterization by cardiac MRI in diagnosing Takotsubo syndrome
}

Pankaj Garg, John P. Greenwood and Sven Plein

We read with great interest the in-depth Review by Akashi et al. (Epidemiology and pathophysiology of Takotsubo syndrome. Nat. Rev. Cardiol. 12, 387-397; 2015). ${ }^{1}$ The authors provide a detailed overview of the diagnostic criteria of Takotsubo cardiomyopathy. We note that the majority of the diagnostic criteria listed by the authors involve traditional methods (including coronary angiography, rise of cardiac enzymes, and regional myocardial dysfunction) to diagnose this form of cardiomyopathy. We would like to point out that a diagnostic strategy based on these criteria alone has the potential to underdiagnose acute coronary syndrome secondary to spontaneous plaque rupture. A study to investigate in vivo coronary histopathological changes by intravascular ultrasonography in individuals diagnosed with Takotsubo syndrome using traditional methods demonstrated plaque rupture of the left anterior descending coronary artery in all five patients. ${ }^{2}$

The diagnostic role of late gadolinium enhancement on cardiac MRI in differentiating between Takotsubo syndrome and myocardial infarction secondary to coronary artery disease is not sufficiently emphasized in the Review. Furthermore, the authors highlight expansion of extracellular matrix in the acute phase of Takotsubo syndrome, which can be characterized by estimation of the extracellular volume fraction from pre/ post contrast $\mathrm{T} 1$ maps using magnetic resonance relaxometry techniques. Cardiac MRI can also provide insights into the various manifestations of Takotsubo syndrome, ${ }^{3}$ which range from a typical pattern of left ventricular regional wall motion abnormality, to apical ballooning, mid-ventricular myocardial dysfunction, and even myocardial dysfunction specific to the coronary artery. ${ }^{4}$ With cardiac MRI, Takotsubo syndrome can be assessed using specific criteria: typical or atypical pattern of left ventricular dysfunction accompanied by myocardial oedema in the dysfunctional zone and absence of clinically significant fibrosis. Although large, multicentre studies are needed to investigative the potential role of cardiac MRI-derived biomarkers to guide treatment or predict prognosis in Takotsubo syndrome, cardiac
MRI, where available, can already assist in the accurate diagnosis of suspected Takotsubo syndrome and the exclusion of other causes of acute coronary syndrome.

Multidisciplinary Cardiovascular Research Centre \& Leeds Institute of Cardiovascular and Metabolic Medicine, University of Leeds, Leeds LS2 9JT, UK (P.G., J.P.G., S.P.).

Correspondence to: S.P.

s.plein@leeds.ac.uk

Competing interests

The authors declare no competing interests.

1. Akashi, Y. J., Nef, H. M. \& Lyon, A. R. Epidemiology and pathophysiology of Takotsubo syndrome. Nat. Rev. Cardiol. 12 , 387-397 (2015).

2. Ibanez, B., Navarro, F., Cordoba, M., M-Alberca, P. \& Farre, J. Tako-tsubo transient left ventricular apical ballooning: is intravascular ultrasound the key to resolve the enigma? Heart 91, 102-104 (2005).

3. Eitel, I. et al. Clinical characteristics and cardiovascular magnetic resonance findings in stress (Takotsubo) cardiomyopathy. JAMA 306, 277-286 (2011).

4. Garg, P., Greenwood, J. P., Plein, S. Multiparametric relaxometry by cardiac magnetic resonance imaging in Takotsubo cardiomyopathy. Eur. Heart J. Cardiovasc. Imaging http:// dx.doi.org/10.1093/ehjci/jev167. 